\title{
Evaluation of a Mixed Meal Test for Diagnosis and Characterization of PancrEaTogEniC DiabeTes Secondary to Pancreatic Cancer and Chronic Pancreatitis: Rationale and Methodology for the DETECT Study From the Consortium for the Study of Chronic Pancreatitis, Diabetes, and Pancreatic Cancer
}

\author{
Phil A. Hart, MD ${ }^{\star}$, Dana K. Andersen, $\mathrm{MD}^{\dagger}$, Kieren J. Mather, MD ${ }^{\ddagger}$, Alicia C. Castonguay, \\ PhD ${ }^{\S}$, , Mandeep Bajaj, MBBS, FRCPI, Melena D. Bellin, MD\#, David Bradley, MD, MS ${ }^{\star *}$, \\ Noemy Contreras, MS§, Aida Habtezion, MD, MSc ${ }^{\dagger \dagger}$, Murray Korc, MD亦, Yogish Kudva,

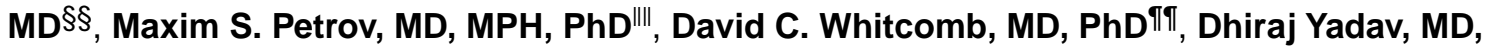 \\ $\mathrm{MPH}^{\# \# \text {, Ying Yuan, PhD§, Jo Ann Rinaudo, PhD }}{ }^{\star \star \star}$, Sudhir Srivastava, PhD, MPH ${ }^{\star \star \star}$, Jose \\ Serrano, MD, $\mathrm{PhD}^{\dagger}$, and on behalf of the Consortium for the Study of Chronic Pancreatitis, \\ Diabetes, and Pancreatic Cancer (CPDPC) ${ }^{\dagger \dagger}$
}

\begin{abstract}
*Division of Gastroenterology, Hepatology, and Nutrition, The Ohio State University Wexner Medical Center, Columbus, $\mathrm{OH}^{\dagger}$ Division of Digestive Diseases and Nutrition, National Institute of Diabetes and Digestive and Kidney Diseases, National Institutes of Health, Bethesda, MD ‡Division of Endocrinology \& Metabolism, Indiana University School of Medicine, Indianapolis IN $\S$ Department of Biostatistics, The University of Texas MD Anderson Cancer Center, Houston, TX "Department of Neurology, University of Toledo, College of Medicine, Toledo, $\mathrm{OH}$ "Section of Endocrinology, Diabetes and Metabolism, Baylor College of Medicine, Houston, TX \#Departments of Pediatrics and Surgery, University of Minnesota Medical School, Minneapolis, MN ${ }^{* *}$ Diabetes and Metabolism Research Center, Division of Endocrinology, Diabetes \& Metabolism, The Ohio State University Wexner Medical Center, Columbus, $\mathrm{OH} \S^{\S}$ Department of Biostatistics, The University of Texas MD Anderson Cancer Center, Houston, TX t+Division of Gastroenterology and Hepatology, Department of Medicine, Stanford University School of Medicine, Stanford, CA ¥¥Departments of Medicine, and Biochemistry and Molecular Biology, Indiana University School of Medicine, the Melvin and Bren Simon Cancer Center, and the Pancreatic Cancer Signature Center, Indianapolis, IN $\S \S$ Division of Endocrinology, Mayo Clinic College of Medicine, Rochester, MN "'IDepartment of Surgery, University of Auckland, Auckland, New Zealand TIDepartments of Medicine, Cell Biology \& Molecular Physiology, and Department of Human Genetics, University of Pittsburgh, and UPMC, Pittsburgh, PA \#\#Division of Gastroenterology \& Hepatology, University of Pittsburgh Medical Center, Pittsburgh, PA §Department of Biostatistics, University of Texas MD Anderson Cancer Center, Houston, TX ${ }^{* *}$ Cancer Biomarker Research Group, Division of Cancer Prevention, National Cancer Institute, National Institutes of Health, Rockville, MD ${ }^{* * *}$ Cancer
\end{abstract}

\footnotetext{
Address correspondence to: Phil A. Hart, MD, Division of Gastroenterology, Hepatology, and Nutrition, The Ohio State University Wexner Medical Center, 410 West Tenth Avenue, Columbus, OH 43210 (philip.hart@ osumc.edu). Phone: 614-366-6213, Fax: 614-293-8518.

Conflicts of interest/disclosures: No conflicts of interest exist.
} 


\author{
Biomarker Research Group, Division of Cancer Prevention, National Cancer Institute, National \\ Institutes of Health, Rockville, MD ${ }^{\dagger}$ Division of Digestive Diseases and Nutrition, National Institute \\ of Diabetes and Digestive and Kidney Diseases, National Institutes of Health, Bethesda, MD \\ ${ }^{+t+}$ Division of Endocrinology, Diabetes and Metabolism, Department of Medicine, Cedars-Sinai \\ Medical Center, Los Angeles, CA
}

\title{
Abstract
}

Pancreatogenic diabetes mellitus is most commonly the result of chronic pancreatitis, but can also occur secondary to pancreatic cancer. The early identification of pancreatogenic diabetes and distinction from the more prevalent type 2 diabetes is clinically significant; however, currently there is no validated method to differentiate these diabetes subtypes. We describe a study, "Evaluation of a Mixed Meal Test for Diagnosis and Characterization of PancrEaTogEniC DiabeTes Secondary to Pancreatic Cancer and Chronic Pancreatitis: the DETECT study," which seeks to address this knowledge gap. The DETECT study is a multicenter study that will examine differences in hormone and glucose excursions following a mixed meal test. The study will also create a biorepository that will be used to evaluate novel diagnostic biomarkers for differentiating these diabetes subtypes.

\section{Keywords}

Type 3c diabetes mellitus; pancreatic polypeptide; insulin; glucagon; incretin hormone

\section{INTRODUCTION}

Diabetes mellitus (DM) secondary to an inherited or acquired disease of the exocrine pancreas is collectively termed pancreatogenic or type $3 \mathrm{c}$ diabetes mellitus (type $3 \mathrm{c} \mathrm{DM}$ ). ${ }^{1,2}$ The most commonly recognized causes of pancreatogenic DM are chronic pancreatitis and pancreatic ductal adenocarcinoma (PDAC), accounting for approximately $80 \%$ and $10 \%$ of cases, respectively (Fig. 1). ${ }^{3}$ The lifetime prevalence of DM in chronic pancreatitis can reach up to $80 \%$, with a point prevalence of $40 \% .^{4,5}$ The prevalence is dependent on both patient and disease-related factors, including age, body mass index, family history, duration of pancreatic disease, presence of exocrine insufficiency, and pancreatic surgery. ${ }^{4,5}$ The prevalence of DM in PDAC at the time of diagnosis is $~ 50 \%$, importantly $75 \%$ of these patients are diagnosed with DM within 2 years prior to the diagnosis of cancer. ${ }^{6}$ The overall prevalence of pancreatogenic DM amongst those with diabetes ranges from 2 to $9 \% .3,7,8$ However, the true prevalence is difficult to determine due to the inability to differentiate it from the more prevalent type $2 \mathrm{DM}$ (T2DM) and lack of validated diagnostic criteria. The primary purpose of the DETECT (Evaluation of a Mixed Meal Test for Diagnosis and Characterization of PancrEaTogEniC DiabeTes Secondary to Pancreatic Cancer and Chronic Pancreatitis) study is to develop a method to distinguish the two aforementioned subtypes of pancreatogenic diabetes from T2DM. 


\section{METABOLIC CHANGES OBSERVED IN PANCREATOGENIC DM}

The pathophysiology of pancreatogenic DM is complex, as defects in insulin secretion, insulin action, and incretin secretion have been previously discussed. ${ }^{2,9}$ The clinical presentation is heterogenous, due to differences in the nature and severity of the underlying pancreatic diseases. A new subclassification of causes of pancreatogenic DM was recently proposed (Table 1) to better distinguish underlying causes. ${ }^{2}$ Considering the known (and suspected) differences in these subtypes of pancreatogenic DM, it is most appropriate to specifically refer to the exocrine pancreatic disease to avoid misinterpretations. For example, herein we refer to two different causes of pancreatogenic DM: 1. Chronic pancreatitisrelated diabetes mellitus (CP-DM), and 2. Pancreatic cancer-related diabetes mellitus (PDAC-DM). This nomenclature is consistent with the widely used designation of cystic fibrosis-related diabetes mellitus (CFRD).

The primary defect in pancreatogenic diabetes is relative or absolute insulin deficiency. Loss of insulin secretion is commonly accompanied by loss of other glucoregulatory islet hormones, including glucagon and pancreatic polypeptide (PP). This global failure of islet hormone release may be mediated by the action of activated pancreatic stellate cells, ${ }^{10}$ the effect of pro-inflammatory cytokines, ${ }^{11}$ or loss of pancreatic parenchyma. In diabetes that is a consequence of, and frequently a harbinger of, PDAC (i.e., PDAC-DM), insulin secretion is impaired due to what is thought to be a paraneoplastic phenomenon, potentially mediated by PDAC-derived exosomes. ${ }^{12}$ Pancreatic polypeptide deficiency might be a unique hallmark of pancreatogenic DM. Particularly in chronic pancreatitis, a deficiency of PP is often seen, and has been associated with reduced hepatic sensitivity to insulin. ${ }^{13}, 14$ Administration of PP reverses hepatic insulin resistance in PP-deficient patients. ${ }^{15-17}$ In PDAC, impaired hepatic insulin sensitivity may be a consequence of the loss of PP secretion associated with tumor localized to the pancreatic head. ${ }^{18,19}$ The primary aim of the DETECT study is to determine whether a deficient PP response to mixed meal stimulation can distinguish pancreatogenic DM from T2DM.

While insulin deficiency is the core defect in pancreatogenic DM, several other abnormalities in glucose homeostasis are frequently observed. Insulin resistance has been documented in both chronic pancreatitis and PDAC. ${ }^{20-23}$ Alpha cell dysfunction (insufficient glucagon response to hypoglycemia and insufficient suppression by oral glucose) was recently found to be similar in subjects with T2DM and CP-DM. ${ }^{24}$ Whether the enteroinsular axis (meal-induced secretion of incretin hormones from the gut that stimulate beta-cell insulin secretion) is disturbed in pancreatogenic DM remains uncertain, given inconsistent results in studies of basal and stimulated incretin hormone levels in chronic pancreatitis with or without diabetes; this axis has not been well studied in PDACDM. ${ }^{25-29}$ In individuals with chronic pancreatitis and impaired glucose tolerance or diabetes and exocrine insufficiency, administration of pancreatic enzyme supplementation boosted GLP-1 and GIP secretion, and consequently the insulin response to liquid meals. ${ }^{30,31}$ The DETECT study is also designed to evaluate insulin resistance, alpha-cell dysfunction and disturbed incretin hormone response in CP-DM and PDAC-DM. 


\section{CLINICAL RELEVANCE OF PANCREATOGENIC DM}

The clinical relevance of pancreatogenic DM differs according to the underlying pancreatic disease. For example, in CP-DM chronic hyperglycemia may lead to long-term complications similar to those observed in T2DM ${ }^{32-34}$ Distinguishing pancreatogenic DM from T2DM impacts treatment given that patients with the former tend to have higher hemoglobin A1c and an earlier need for insulin. ${ }^{7}$ In the absence of clinical trials, treatment approaches for pancreatogenic DM have been extrapolated from management of type $1 \mathrm{DM}$ (T1DM) and T2DM. ${ }^{2,9}$

In addition to the need to treat hyperglycemia and avoid long-term complications, the presence of pancreatogenic DM may present a unique opportunity for early diagnosis of PDAC. Importantly, studies have demonstrated a markedly increased risk for developing PDAC in those diagnosed with CP-DM..$^{35,36}$ On the other hand, new onset diabetes occurs in up to $50 \%$ of patients with PDAC, with many more having glucose intolerance. ${ }^{37,38}$ The PDAC-DM diagnosis typically occurs from 6 months to three years before the PDAC is diagnosed, and roughly half of the patients with new-onset diabetes show improvement or resolution of hyperglycemia either following resection of the malignancy or treatment response to neoadjuvant chemotherapy. ${ }^{6,38-40}$ Thus, the development of hyperglycemia may represent the earliest "symptom" of PDAC, providing a potential window of opportunity for early detection. However, historical and clinical features do not clearly differentiate patients with PDAC-DM from patients with new-onset T2DM. ${ }^{41}$ Therefore, a key secondary purpose of the DETECT study is to provide a platform for evaluation of novel protein and peptide markers as indicators of new onset PDAC-DM compared to T2DM.

\section{CURRENT DIAGNOSTIC APPROACH TO PANCREATOGENIC DM}

In cases of antecedent chronic pancreatic disease, the subsequent development of diabetes is highly likely to be pancreatogenic. ${ }^{42}$ In contrast, in cases of new onset diabetes in the absence of known pancreatic disease, pancreatogenic DM is diagnosed clinically following exclusion of T1DM (ruled out by the absence of antibodies associated with T1DM)) and identification of a functional or imaging abnormalities associated with a pancreatic disease. Rickels et al suggested that the disparity of PP levels, which are elevated in T2DM and low in various subtypes of pancreatogenic DM, might confirm the presence of pancreatogenic DM. ${ }^{43}$ Low basal and nutrient-stimulated PP levels have been reported in CP-DM, pancreatic resection, cystic fibrosis, and PDAC-DM, all of these studies have been in small cohorts of patients and need to be further validated in the disease subgroups. ${ }^{9,18,44}$ The need for validated diagnostic criteria for pancreatogenic DM is urgent in order to advance clinical studies.

In 2013, the National Institute of Diabetes and Digestive and Kidney Diseases (NIDDK) and the National Cancer Institute (NCI) sponsored a workshop in which many research needs were identified, including the need to differentiate pancreatogenic DM from T2DM. ${ }^{45}$ This workshop and the passage of the Recalcitrant Cancer Act by the US Congress in 2012 provided the justification for the development of a program co-sponsored and jointly funded by the NIDDK and NCI that is now called the Consortium for the Study of Pancreatitis, 
Diabetes, and Pancreatic Cancer (CPDPC) ${ }^{46,47}$ The Type 3c (pancreatogenic) Diabetes Working Group was chartered soon after the organization of the CPDPC. The DETECT study is the primary study of this working group.

\section{STUDY DESIGN}

\section{Hypotheses and Objectives}

The general hypothesis underlying the DETECT study is that hormone and glucose excursions measured during a mixed meal test can distinguish patients with new onset PDAC-DM and/or CP-DM from patients with T2DM. For the purposes of this study, new onset diabetes is defined as diabetes that has developed within the preceding 36 months. Our primary objective is to test the hypothesis that the relative change in PP levels at 30 minutes following meal stimulation will be significantly lower (defined a priori as $20 \%$ lower) in the PDAC-DM and CP-DM groups compared to T2DM. Secondary objectives include additional comparisons across the same groups regarding insulin, glucagon, and incretin hormone responses.

\section{Study Design Summary}

\section{Enrollment}

The goal of the study is to recruit a total of 452 subjects with PDAC, chronic pancreatitis, and no structural pancreatic disease (Table 2) at 9 participating clinical centers. Control subjects will be considered to have "no structural pancreatic disease" in the absence of clinical, laboratory, and/or imaging data to support the diagnosis of a pancreatic disorder (i.e., chronic pancreatitis or PDAC). Eligible subjects will be approached through a variety of methods, including participation in other related clinical studies performed by the CPDPC. ${ }^{48,49}$

\section{Mixed Meal Testing Protocol}

To reduce confounding from medication use, the following medications will be held on the morning of the study visit: antihyperglycemic agents, pancreatic enzyme supplementation, and systemic anticholinergics. Subjects will be asked to consume a standard mixed meal consisting of $12 \mathrm{oz}$ Boost ${ }^{\circledR}$ drink, chocolate flavor providing 360 calories, $15 \mathrm{~g}$ protein, 61.5 $\mathrm{g}$ carbs, and $6 \mathrm{~g}$ fat. Blood samples will be collected at fasting baseline ( -5 and 0 minutes), then 15, 30, 45, 60, 90, and 120 minutes following meal stimulation. Samples will be processed and stored according to a study-specific biospecimen standard operating procedures manual. The samples will be batch analyzed at a central laboratory to measure the analytes of interest, including PP, glucose, c-peptide, insulin, glucagon, GLP-1, and GIP levels. All subjects will complete a standardized questionnaire regarding personal medical and medication histories as well as family history. 


\section{Eligibility Criteria}

\section{Inclusion Criteria}

Subjects with PDAC, chronic pancreatitis, and no disease of the exocrine pancreas will be enrolled based on their diabetes status as illustrated in Table 2. Subjects must be ages $\geq 40$ and $<85$ years. The rationale for the age restriction is to concentrate the study population to an age distribution that overlaps with both PDAC and chronic pancreatitis.

\section{Exclusion Criteria}

Subjects using high dosages of insulin (total daily dose $\searrow 0.5$ unit/kg/day), long-acting antihyperglycemic agents, or other medications that would confound the study results will not be enrolled. Similarly, subjects with a prior pancreatic surgery (including total pancreatectomy, pancreaticoduodenectomy, distal pancreatectomy, pancreaticojejunostomy, enucleation, or Frey procedure), gastric surgery, or vagotomy are ineligible. Lastly, subjects with PDAC who have received previous treatment for pancreatic cancer, including chemotherapy or radiation, will be excluded.

\section{Statistical Power Calculation}

The priority for the test being studied is to rule out T2DM in patients who may have T2DM, PDAC-DM, or CP-DM; therefore, a high sensitivity is desirable. Fixing the sensitivity at $90 \%$, the mixed meal test is regarded as not useful if its specificity to distinguish new-onset T2DM from PDAC-DM or CP-DM is $25 \%$. Based on preliminary data examining PP response for diagnosis of pancreatogenic DM (in subjects with new onset PDAC-DM and T2DM), ${ }^{19}$ when the sensitivity is $90 \%$, the specificity is approximately $44 \%$. If we assume that the true specificity for this study is $44 \%$ and the significance level of $5 \%$, to achieve $80 \%$ power, we need to enroll 136 subjects with new onset PDAC-DM, 136 subjects with new onset CP-DM, and 100 subjects with new onset T2DM (Table 2). A smaller number of subjects (10-20 per subgroup) will be enrolled to generate pilot data from the six subject sub-groups with either long-standing diabetes or no diabetes.

\section{Statistical Plan}

The Wilcoxon signed-rank test and $t$-test will be used to determine if there is a significant difference in the PP response (measured as the change of absolute and relative levels of PP compared to fasting, basal levels) at each time point (with the primary outcome at 30 minutes). Intergroup analyses will be performed to compare the median (or mean) relative change in PP (\%) from baseline to each time point, as well as the area under the curves (AUC), using the Kruskal-Wallis test and analysis of variance (ANOVA). Linear regression and mixed model will be conducted to further compare the changes in PP (\%) from baseline between three new onset diabetes subgroups at each time point and across time points, while controlling potential confounders. Comparison of the groups based on insulin, glucagon, and incretin secretion responses will be executed using a similar statistical methodology. 


\section{FUTURE IMPACT OF THE DETECT STUDY}

The DETECT study is the first to comprehensively compare dynamic changes in beta-cell function, insulin resistance, and pancreatic and gut hormone levels using a standardized meal in a large cohort of subjects with T2DM, PDAC-DM, and CP-DM. These data will be useful to develop diagnostic criteria for pancreatogenic DM, refine our understanding and ongoing studies of CP-DM, and further examine the potential to screen for sporadic PDAC. The results will be used to propose diagnostic criteria for pancreatogenic DM for use in both future research studies and clinical practice. Such criteria are awaited to execute more rigorous epidemiologic studies to refine the estimates of the burden of pancreatogenic DM. Furthermore, the ability to diagnose pancreatogenic DM will guide management decisions and foster prospective studies to define the role of such testing in predicting the risk of DM and whether intervention(s) can help to delay its onset. Furthermore, data generated from the DETECT study will also allow ancillary comparisons within the cohort studies being performed within the CPDPC, particularly in chronic pancreatitis. Stratification of chronic pancreatitis subjects in Prospective Evaluation of Chronic Pancreatitis for Epidemiologic and Translational Studies (PROCEED) by the type of DM based on differential glucose and hormone responses will also be used to assess different patterns of risk for disease-related outcomes, including exocrine insufficiency, metabolic bone disease, and interval PDAC. ${ }^{49}$ Finally, accurate discrimination of PDAC-DM from the much more prevalent T2DM amongst patients with undifferentiated diabetes would define a high-risk group in whom further screening studies would be feasible.

The DETECT study specimen collections are carefully planned to allow future evaluation of both baseline and meal-stimulated biomarkers. It is unclear if there are host-related biomarkers (such as genetic markers) that are distinct for pancreatogenic DM compared to T1DM and T2DM and what degree of heterogeneity exists within the various subtypes of pancreatogenic DM. For example, is pancreatogenic DM related to chronic pancreatitis different from the diabetes related to all PDAC, or to subsets of PDAC? We envision the current and future biomarker studies using high-throughput assays (including genomic, proteomic, and metabolomics approaches) from the DETECT study will provide similar insights to those that have occurred in other chronic diseases, including T2DM, to allow investigators to monitor, predict, and unravel mechanisms of disease pathogenesis.

In summary, the DETECT study is a large, multicenter clinical study that seeks to identify a simple clinical test to distinguish new onset PDAC-DM and CP-DM from the more common T2DM. The study will involve collection of biosamples prior to and following mixed meal stimulation to comprehensively assess different patterns of beta-cell function, insulin resistance, and pancreatic and gut hormone responses. These results will provide a strong foundation to support ongoing and future studies to further understand the underlying pathophysiology, diagnosis, and treatment of pancreatogenic DM.

\section{Grant Support:}

Research reported in this publication was supported by the National Cancer Institute and National Institute of Diabetes and Digestive and Kidney Diseases (NIDDK) under award numbers: U01DK108327 (PH, Bellin, DB), U01DK108288 (YK), U01DK108323 (KM, MK), U01DK108326 (Bajaj), U01DK108328 (AC, NC, YY), 
U01DK108300 (AH), U01DK108314 (MP, MG), and U01DK108306 (DCW, DY). The content is solely the responsibility of the authors and does not necessarily represent the official views of the National Institutes of Health.

\section{Abbreviations:}

$\begin{array}{ll}\text { DM } & \text { diabetes mellitus } \\ \text { PDAC } & \text { pancreatic ductal adenocarcinoma } \\ \text { PP } & \text { pancreatic polypeptide } \\ \text { T1DM } & \text { type } 1 \text { diabetes mellitus } \\ \text { T2DM } & \text { type } 2 \text { diabetes mellitus } \\ \text { T3cDM } & \text { type } 3 c \text { diabetes mellitus }\end{array}$

\section{REFERENCES}

1. Report of the expert committee on the diagnosis and classification of diabetes mellitus. Diabetes Care. 1997;20:1183-1197. [PubMed: 9203460]

2. Hart PA, Bellin MD, Andersen DK, et al. Type 3c (pancreatogenic) diabetes mellitus secondary to chronic pancreatitis and pancreatic cancer. Lancet Gastroenterol Hepatol. 2016;1:226-237. [PubMed: 28404095]

3. Ewald N, Kaufmann C, Raspe A, et al. Prevalence of diabetes mellitus secondary to pancreatic diseases (type 3c). Diabetes Metab Res Rev. 2012;28:338-342. [PubMed: 22121010]

4. Malka D, Hammel P, Sauvanet A, et al. Risk factors for diabetes mellitus in chronic pancreatitis. Gastroenterology. 2000;119:1324-1332. [PubMed: 11054391]

5. Bellin MD, Whitcomb DC, Abberbock J, et al. Patient and disease characteristics associated with the presence of diabetes mellitus in adults with chronic pancreatitis in the United States. Am J Gastroenterol. 2017;112:1457-1465. [PubMed: 28741615]

6. Pannala R, Leirness JB, Bamlet WR, et al. Prevalence and clinical profile of pancreatic cancerassociated diabetes mellitus. Gastroenterology. 2008;134:981-987. [PubMed: 18395079]

7. Woodmansey C, McGovern AP, McCullough KA, et al. Incidence, demographics, and clinical characteristics of diabetes of the exocrine pancreas (Type 3c): A retrospective cohort study. Diabetes Care. 2017;40:1486-1493. [PubMed: 28860126]

8. Pendharkar SA, Mathew J, Petrov MS. Age- and sex-specific prevalence of diabetes associated with diseases of the exocrine pancreas: A population-based study. Dig Liver Dis. 2017;49:540-544. [PubMed: 28110921]

9. Cui Y, Andersen DK. Pancreatogenic diabetes: special considerations for management. Pancreatology. 2011;11:279-294. [PubMed: 21757968]

10. Kikuta K, Masamune A, Hamada S, et al. Pancreatic stellate cells reduce insulin expression and induce apoptosis in pancreatic beta-cells. Biochem Biophys Res Commun. 2013;433:292-7. [PubMed: 23500461]

11. Pondugala PK, Sasikala M, Guduru VR, et al. Interferon-gamma decreases nuclear localization of Pdx-1 and triggers beta-cell dysfunction in chronic pancreatitis. J Interferon Cytokine Res. 2015;35:523-529. [PubMed: 25839229]

12. Javeed N, Sagar G, Dutta SK, et al. Pancreatic cancer-derived exosomes cause paraneoplastic betacell dysfunction. Clin Cancer Res. 2015;21:1722-1733. [PubMed: 25355928]

13. Sun YS, Brunicardi FC, Druck P, et al. Reversal of abnormal glucose metabolism in chronic pancreatitis by administration of pancreatic polypeptide. Am J Surg. 1986;151:130-140. [PubMed: 3946744] 
14. Seymour NE, Volpert AR, Lee EL, et al. Alterations in hepatocyte insulin binding in chronic pancreatitis: effects of pancreatic polypeptide. Am J Surg. 1995;169:105-109; discussion 110. [PubMed: 7817978]

15. Seymour NE, Brunicardi FC, Chaiken RL, et al. Reversal of abnormal glucose production after pancreatic resection by pancreatic polypeptide administration in man. Surgery. 1988;104:119-129. [PubMed: 3041640]

16. Brunicardi FC, Chaiken RL, Ryan AS, et al. Pancreatic polypeptide administration improves abnormal glucose metabolism in patients with chronic pancreatitis. J Clin Endocrinol Metab. 1996;81:3566-3572. [PubMed: 8855802]

17. Rabiee A, Galiatsatos P, Salas-Carrillo R, et al. Pancreatic polypeptide administration enhances insulin sensitivity and reduces the insulin requirement of patients on insulin pump therapy. $\mathrm{J}$ Diabetes Sci Technol. 2011;5:1521-1528. [PubMed: 22226275]

18. Skrha J, Busek P, Uhrova J, et al. Lower plasma levels of glucose-dependent insulinotropic peptide (GIP) and pancreatic polypeptide (PP) in patients with ductal adenocarcinoma of the pancreas and their relation to the presence of impaired glucoregulation and weight loss. Pancreatology. 2017; 17:89-94. [PubMed: 28027898]

19. Hart PA, Baichoo E, Bi Y, et al. Pancreatic polypeptide response to a mixed meal is blunted in pancreatic head cancer associated with diabetes mellitus. Pancreatology. 2015;15:162-166. [PubMed: 25766398]

20. Niebisz-Cieślak AB, Karnafel W. Insulin sensitivity in chronic pancreatitis and features of insulin resistance syndrome. Pol Arch Med Wewn. 2010;120:255-263. [PubMed: 20693955]

21. Vlasáková ZL, Bartos V, Spicák J [Diabetes mellitus in chronic pancreatitis and insulin sensitivity]. [Article in Czech]. Vnitr Lek. 2002;48:878-881. [PubMed: 16737129]

22. Yki-Järvinen H, Kiviluoto T, Taskinen MR. Insulin resistance is a prominent feature of patients with pancreatogenic diabetes. Metabolism. 1986;35:718-727. [PubMed: 3736412]

23. Cersosimo E, Pisters PW, Pesola G, et al. Insulin secretion and action in patients with pancreatic cancer. Cancer. 1991;67:486-493. [PubMed: 1985741]

24. Mumme L, Breuer TGK, Rohrer S, et al. Defects in alpha-cell function in patients with diabetes due to chronic pancreatitis compared with patients with type 2 diabetes and healthy individuals. Diabetes Care. 2017;40:1314-1322. [PubMed: 28751547]

25. Hornum M, Pedersen JF, Larsen S, et al. Increased postprandial response of glucagon-like peptide-2 in patients with chronic pancreatitis and pancreatic exocrine insufficiency. Pancreatology. 2010;10:201-207. [PubMed: 20460948]

26. Knop FK, Vilsbøll T, Højberg PV, et al. Reduced incretin effect in type 2 diabetes: cause or consequence of the diabetic state? Diabetes. 2007;56:1951-1959. [PubMed: 17513701]

27. Gómez-Cerezo J, Garcés MC, Codoceo R, et al. Postprandial glucose-dependent insulinotropic polypeptide and insulin responses in patients with chronic pancreatitis with and without secondary diabetes. Regul Pept. 1996;67:201-205. [PubMed: 8988521]

28. Hiroyoshi M, Tateishi K, Yasunami Y, et al. Elevated plasma levels of glucagon-like peptide-1 after oral glucose ingestion in patients with pancreatic diabetes. Am J Gastroenterol. 1999;94:976-81. [PubMed: 10201468]

29. Jensen SL, Lauridsen KB, Christensen KC, et al. Diminished gastric inhibitory polypeptide response to glucose administered orally in patients with chronic pancreatitis. Surg Gynecol Obstet. 1981;153:665-668. [PubMed: 7027498]

30. Ebert R, Creutzfeldt W. Reversal of impaired GIP and insulin secretion in patients with pancreatogenic steatorrhea following enzyme substitution. Diabetologia. 1980;19:198-204. [PubMed: 6997121]

31. Knop FK, Vilsbøll T, Larsen S, et al. Increased postprandial responses of GLP-1 and GIP in patients with chronic pancreatitis and steatorrhea following pancreatic enzyme substitution. Am J Physiol Endocrinol Metab. 2007;292:E324-E330. [PubMed: 16954337]

32. Briani G, Riva F, Midena E, et al. Prevalence of microangiopathic complications in hyperglycemia secondary to pancreatic disease. J Diabet Complications. 1988;2:50-52. [PubMed: 2968358]

33. Ziegler O, Candiloros H, Guerci B, et al. Lower-extremity arterial disease in diabetes mellitus due to chronic pancreatitis. Diabete Metab. 1994;20:540-545. [PubMed: 7713277] 
34. Couet C, Genton P, Pointel JP, et al. The prevalence of retinopathy is similar in diabetes mellitus secondary to chronic pancreatitis with or without pancreatectomy and in idiopathic diabetes mellitus. Diabetes Care. 1985;8:323-328. [PubMed: 4042797]

35. Liao KF, Lai SW, Li CI, et al. Diabetes mellitus correlates with increased risk of pancreatic cancer: a population-based cohort study in Taiwan. J Gastroenterol Hepatol. 2012;27:709-713. [PubMed: 21929650]

36. Brodovicz KG, Kou TD, Alexander CM, et al. Impact of diabetes duration and chronic pancreatitis on the association between type 2 diabetes and pancreatic cancer risk. Diabetes Obes Metab. 2012;14:1123-1128. [PubMed: 22831166]

37. Cui Y, Andersen DK. Diabetes and pancreatic cancer. Endocr Relat Cancer. 2012;19:F9-F26. [PubMed: 22843556]

38. Pannala R, Basu A, Petersen GM, et al. New-onset diabetes: a potential clue to the early diagnosis of pancreatic cancer. Lancet Oncol. 2009;10:88-95. [PubMed: 19111249]

39. Gardner TB, Hessami N, Smith KD, et al. The effect of neoadjuvant chemoradiation on pancreatic cancer-associated diabetes mellitus. Pancreas. 2014;43:1018-1021. [PubMed: 25000339]

40. Permert J, Ihse I, Jorfeldt L, et al. Improved glucose metabolism after subtotal pancreatectomy for pancreatic cancer. Br J Surg. 1993;80:1047-1050. [PubMed: 8402064]

41. Chari ST, Leibson CL, Rabe KG, et al. Pancreatic cancer-associated diabetes mellitus: prevalence and temporal association with diagnosis of cancer. Gastroenterology. 2008;134:95-101. [PubMed: 18061176]

42. Ewald N, Bretzel RG. Diabetes mellitus secondary to pancreatic diseases (Type 3c)--are we neglecting an important disease? Eur J Intern Med. 2013;24:203-206. [PubMed: 23375619]

43. Rickels MR, Bellin M, Toledo FG, et al. Detection, evaluation and treatment of diabetes mellitus in chronic pancreatitis: recommendations from PancreasFest 2012. Pancreatology. 2013;13:336-342. [PubMed: 23890130]

44. Nagpal SJS, Bamlet WR, Kudva YC, et al. Comparison of fasting human pancreatic polypeptide levels among patients with pancreatic ductal adenocarcinoma, chronic pancreatitis, and type 2 diabetes mellitus. Pancreas. 2018;47:738-741. [PubMed: 29771765]

45. Andersen DK, Andren-Sandberg $\AA$, Duell EJ, et al. Pancreatitis-diabetes-pancreatic cancer: summary of an NIDDK-NCI workshop. Pancreas. 2013;42:1227-37. [PubMed: 24152948]

46. Serrano J, Andersen DK, Forsmark CE, et al. Consortium for the Study of Chronic Pancreatitis, Diabetes, and Pancreatic Cancer: From Concept to Reality. Pancreas. 2018;47:\#\#\#-\#\#\#\#.

47. Pandol SJ, Forsmark CE, Hart PA, et al. Acceleration of our understanding of recurrent acute and chronic pancreatitis. Pancreatology. 2016;16:692-693. [PubMed: 27542963]

48. Maitra A, Sharma A, Brand RE, et al. A Prospective Study to Establish a New-Onset Diabetes Cohort: From the Consortium for the Study of Chronic Pancreatitis, Diabetes, and Pancreatic Cancer. Pancreas. 2018;47:\#\#\#\#-\#\#\#.

49. Yadav D, Park WG, Fogel E, et al. PROspective Evaluation of Chronic Pancreatitis for EpidEmiologic and Translational StuDies (PROCEED): Rationale and Study Design From the Consortium for the Study of Chronic Pancreatitis, Diabetes, and Pancreatic Cancer. Pancreas. 2018;47:\#\#\#\#-\#\#\#\#. 


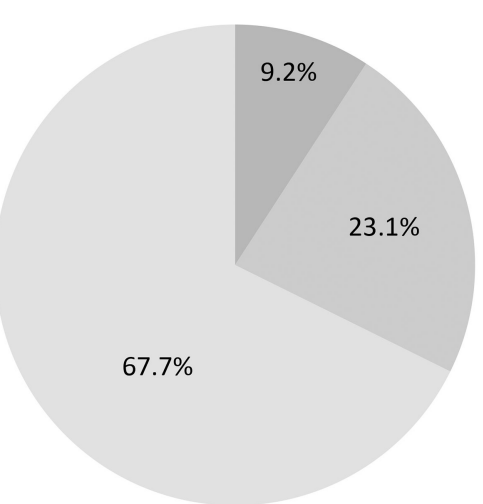

A

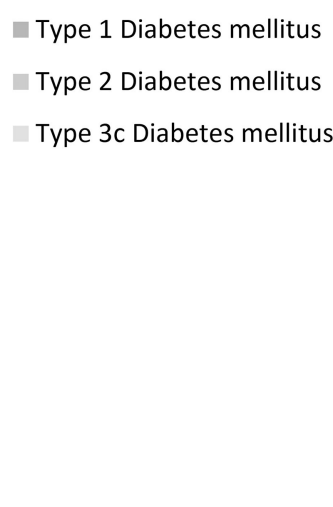

B

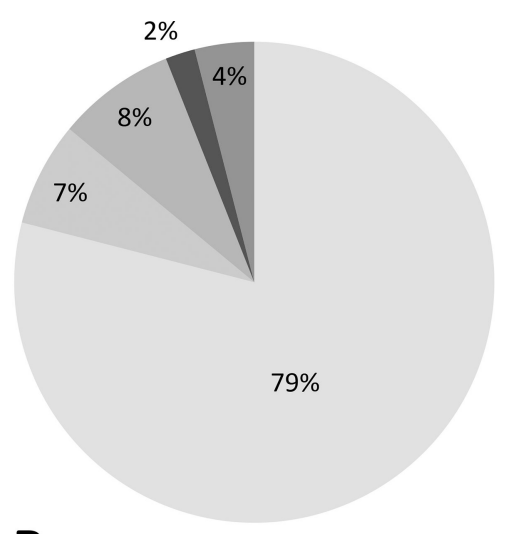

\author{
Chronic Pancreatitis \\ Hereditary Hemochromatosis \\ - Pancreatic Cancer \\ - Status post Pancreatic Resection \\ Cystic Fibrosis
}

FIGURE 1.

Prevalence and etiologies of pancreatogenic DM. A, Prevalence of pancreatogenic (Type 3c) DM in a cohort of 1868 subjects with diabetes. B, Proportion of different subtypes in the subset of subjects $(\mathrm{n}=117)$ with pancreatogenic DM. Adapted with permission. ${ }^{3}$ 
Table 1.

Proposed Subclassification of Pancreatogenic Diabetes According to Their Potential Mechanisms

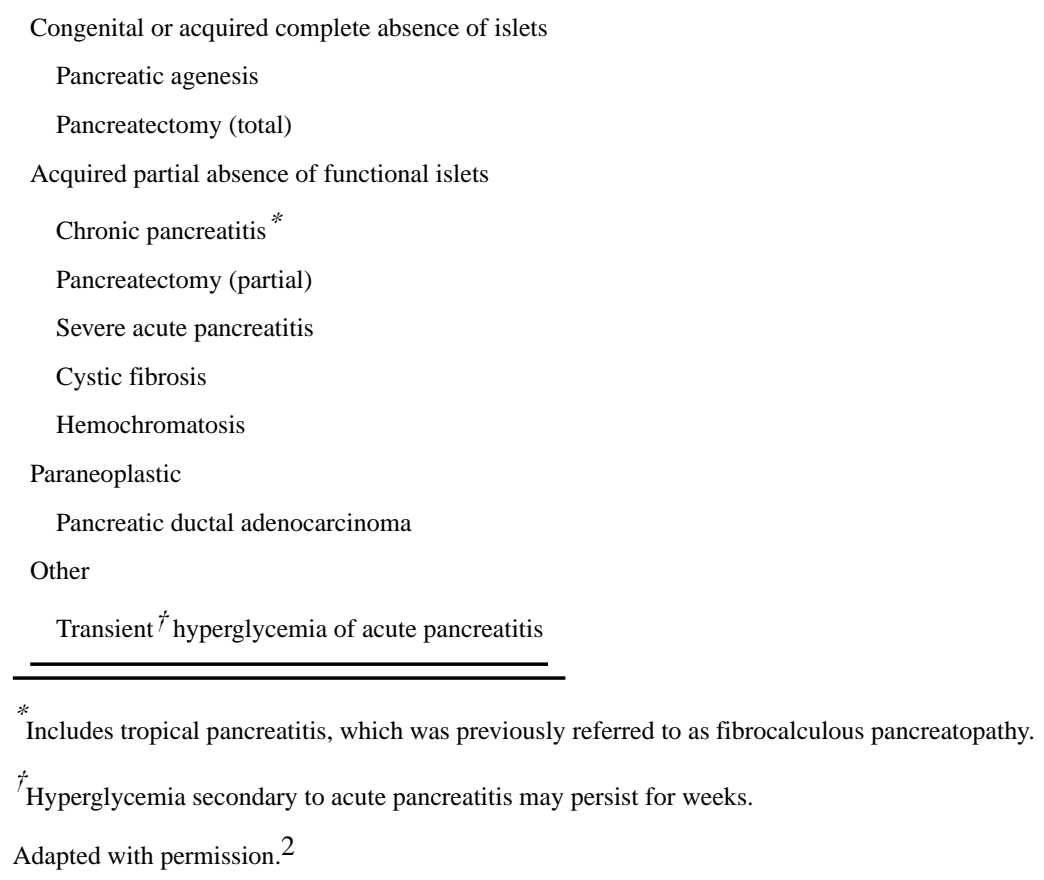


Table 2.

DETECT Study Cohort Composition

\begin{tabular}{lccc}
\hline Pancreatic Disease State & \multicolumn{3}{c}{ Diabetes Status, $\mathbf{~}$} \\
\cline { 2 - 4 } & New-onset & Long-standing & Non-diabetic \\
\hline No Disease & $100^{*}$ & $10^{*}$ & $10^{\dagger}$ \\
Chronic Pancreatitis & 136 & 10 & 10 \\
Pancreatic Cancer & 136 & 20 & 20 \\
\hline
\end{tabular}

Indicates subjects with T2DM.

${ }^{\dagger}$ Indicates healthy control subjects without diabetes. 